\title{
Apoptosis and disease progression in the spontaneously diabetic $\mathrm{BB} / \mathrm{S}$ rat
}

\author{
F. J. Lally, H. Ratcliff, A.J. Bone \\ School of Pharmacy and Biomolecular Sciences, University of Brighton, Brighton, UK
}

\section{Abstract}

Aims/hypothesis. Type I (insulin-dependent) diabetes mellitus is an autoimmune disease culminating in pancreatic beta-cell destruction. A role for apoptosis in this destruction has been suggested, although controversy exists over the identity of the apoptotic cells and the time of onset of apoptosis. This study investigates the extent and timing of islet cell apoptosis in vivo in the spontaneously diabetic $\mathrm{BB} / \mathrm{S}$ rat.

Methods. Pancreatic biopsies were taken from 30 diabetes-prone and 6 diabetes-resistant $\mathrm{BB} / \mathrm{S}$ rats matched for age. Animals were serially biopsied before, during and after development of diabetes and apoptotic cells analysed in serial sections. The diabetesprone group included animals $(n=6)$ that had insulitis but did not develop diabetes.

Results. Apoptosis was not detected in any pancreatic sections from diabetes resistant animals at any age investigated or from any animal before 50 days of age. By 68 days, apoptosis was, however, detectable in both the diabetes-prone group and in the group that had insulitus but did not develop diabetes and this correlated with a decrease in pancreatic insulin staining and a development of insulitis. There was a fur- ther increase in apoptosis in the diabetes-prone group at 85 days, which coincided with the time of onset of diabetes ( 84 days). In addition, there was a sixfold increase in intra-islet apoptosis between 68 and 85 days in the diabetes-prone group and at 85 days intra-islet apoptosis was threefold higher in the diabetes-prone group than in the group that had insulitus but did not develop diabetes. At 107 days, apoptosis (total and intra-islet) was higher in the group that had insulitus but did not develop diabetes (OND-DP) than in either the diabetes resistant (DR) or diabetes-prone (DP) groups.

Conclusion/interpretation. We have shown significant islet cell apoptosis in the pancreas of diabetes-prone $\mathrm{BB} / \mathrm{S}$ rats, which coincides with the appearance of insulitis and the onset of diabetes. We have also detected differences in the levels of apoptosis between diabetic and non-diabetic animals and suggest that such differences could be an important determinant of disease progression in this animal model of Type I diabetes. [Diabetologia (2001) 44: 320-324]

Keywords Apoptosis, BB/S rat; Type I diabetes mellitus, immunohistochemistry, insulin.
The pathogenic mechanisms underlying progression to Type I (insulin-dependent) diabetes mellitus are

Received: 15 May 2000 and in revised form: 31 October 2000

Corresponding author: A.J. Bone, School of Pharmacy and Biomolecular Sciences, University of Brighton, Lewes Road, Moulsecoomb, Brighton, East Sussex, BN2 4GJ, UK

Abbreviations: BB/S, Bio-Breeding rat/Southampton colony; DR, diabetes-resistant; DP, diabetes-prone; OND-DP, old non-diabetic diabetes-prone; DAB, diaminobenzidine. not clear but a role of apoptosis in beta-cell destruction has been suggested. Eukaryotic cells can die by either necrosis or apoptosis. Necrosis is a pathological condition, typified by cell swelling and the release of cellular components into the extracellular matrix. In contrast, apoptosis is an ordered process that plays a key part in the regulation of tissue homeostasis and development and is characterised by cell shrinkage, nuclear condensation and, eventually, by the phagocytosis of the apoptotic cell [1]. 


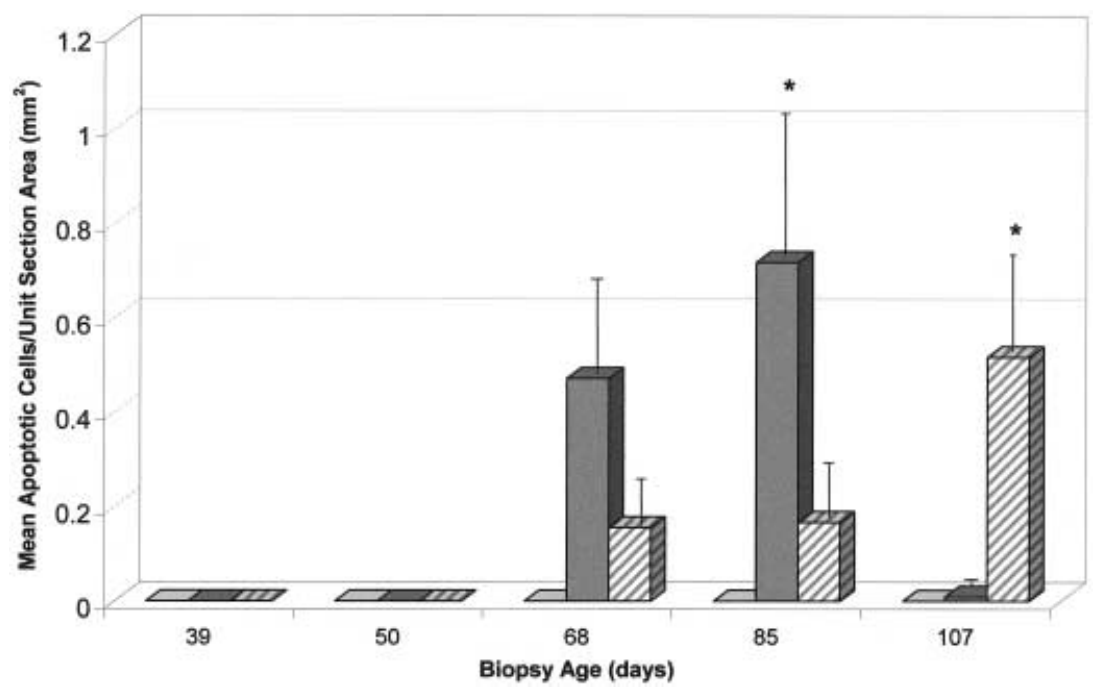

Fig. 1. Total number of apoptotic cells/unit section area $\left(\mathrm{mm}^{2}\right)$. The number of apoptotic cells in sections from DR $(\square)$, DP $(\square)$ and OND-DP (Z) animals were counted and expressed for each unit area $\left(\mathrm{mm}^{2}\right)$. Results are expressed as means \pm SEM. Data were analysed using Kruskal-Wallis analysis and the Mann-Whitney U-test and differences considered significant if the $p$ value was less than 0.05 . $* p<0.05$ vs DR.

Pancreatic islets of Langerhans and beta-cell lines have been shown to undergo apoptosis in vitro in response to cellular stress [2]. Apoptosis of pancreatic beta-cells in vivo, however, is less well documented. The process of apoptosis is so rapid that quantifying apoptosis in vivo is extremely difficult. Despite this, studies in the non-obese diabetic (NOD) mouse model have implicated apoptosis in the mechanism of beta-cell destruction in Type I diabetes mellitus $[3,4]$. There is, however, still considerable debate about the precise timing and extent of apoptosis.

The spontaneously diabetic Bio-Breeding (BB) rat is a good experimental model for the study of autoimmune diabetes. The Southampton $(\mathrm{BB} / \mathrm{S})$ colony consists of two sub-lines: diabetes-resistant (DR) animals that have been diabetes-free for more than 17 generations; diabetes-prone (DP) animals that show an $82 \%$ incidence of diabetes, with onset at 75 to 90 days of age. About $18 \%$ of diabetes-prone animals, although exhibiting insulitis, do not develop overt diabetes (OND-DP group) [5]. By serial pancreatic biopsies on individual animals before, during and after onset of diabetes we can precisely record cellular changes within the pancreas and correlate these with disease progression. Most importantly, however, it also allows us to document any differences between the diabetes-prone and OND-diabetesprone animals.

The aim of this study was to examine apoptosis in pancreatic sections from the spontaneously diabetic
$\mathrm{BB} / \mathrm{S}$ rat and, by comparing results from $\mathrm{DR}$, diabetes-prone and OND-diabetes-prone animals, to investigate the hypothesis that resistance to diabetes involves an alteration in the extent of beta-cell apoptosis.

\section{Methods}

Animals. BB/S rats were obtained from the authors' breeding colony maintained at the University of Southampton. The animals were housed at a constant temperature $\left(18^{\circ} \mathrm{C}\right)$ and humidity $(45 \%)$ on a 12 -h light/dark cycle. They had free access to standard laboratory rat chow and tap water. All experiments were carried out according to the rules and regulations laid down by the Home Office (Animal Scientific Procedure Act 1986, UK). We used DP $(n=30)$ and DR animals matched for age $(n=6)$ from 4 cohorts. Of the diabetes-prone animals, 6 did not develop diabetic symptoms and were classified as OND-diabetes-prone rats. Diabetes was confirmed on the basis of body weight loss, glycosuria (Multistix, Ames UK) and a blood glucose estimation (Accutrend, BCL, UK) of more than $11 \mathrm{mmoll}^{-1}$.

Serial pancreatic biopsies. Pancreatic biopsies were carried out on each animal at 39, 50, 68, 85 and 107 days of age. This procedure was done under aseptic conditions as described previously [6]. Briefly, the rats were anaesthetised with halothane and a small portion of the pancreas was excised. The excised tissue was immediately fixed in $10 \%$ buffered formalin. After the peritoneal and abdominal cavities were closed, the animals were given pure oxygen and subcutaneous Temgesic $\left(0.5 \mathrm{mgKg}^{-1}\right)$ for pain relief. All animals were observed until fully recovered. This procedure does not alter the course of disease progression in this animal model [6].

Immunohistochemical analysis. Formalin-fixed tissues were blocked in paraffin. Sections were cut at $2 \mu \mathrm{m}$ and mounted onto vectabond-treated slides that were dried at $60^{\circ} \mathrm{C}$ for $30 \mathrm{~min}$. Sections were de-waxed and endogenous peroxidase activity blocked by immersing the slides in $3 \%$ hydrogen peroxide in methanol. Tissue sections for apoptosis staining were then permeabilised by incubation with proteinase $K$ $\left(20 \mu \mathrm{gml}^{-1}\right)$. The DeadEnd colorimetric system (Promega, 


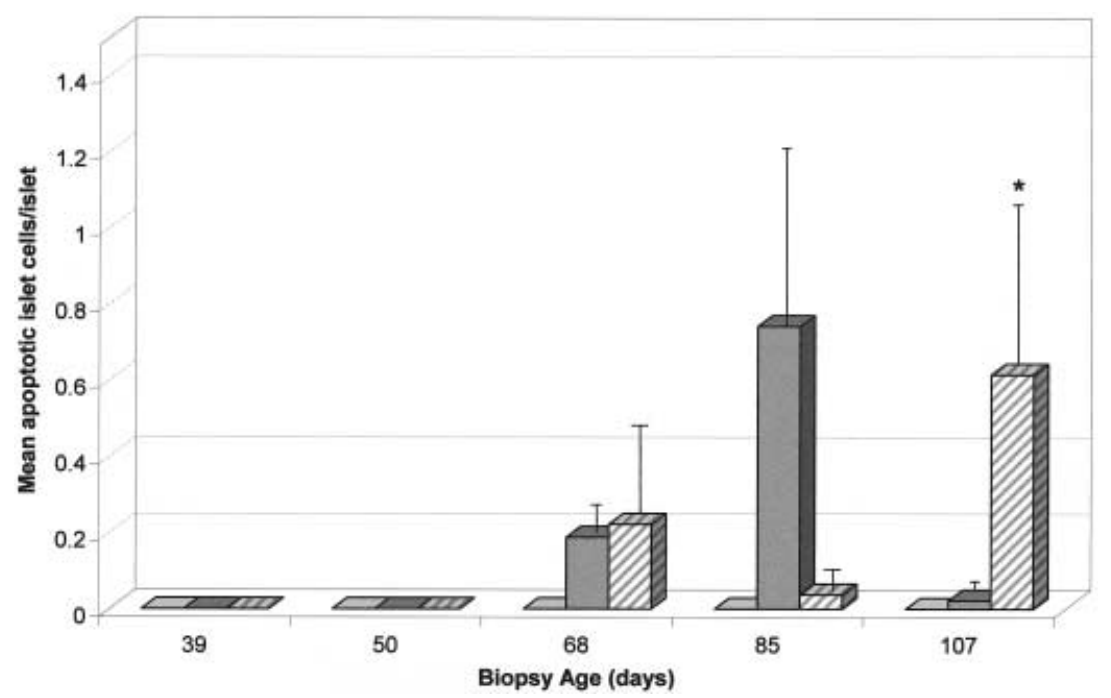

Fig. 2. The number of apoptotic cells occurring within islets in

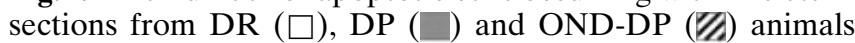
were counted and expressed per islet. Results are expressed as means \pm SEM. Data were analysed using Kruskal-Wallis analysis and differences deemed significant if $p<0.05$. $* p<0.05$ vs DR.

Southampton, UK) was used for apoptosis detection. The sections were background-stained with haematoxylin and mounted. Cells undergoing apoptosis were identified by the presence of DAB staining and apoptotic artefacts (chromatin condensation, apoptotic bodies) using light and confocal microscopy. The total number of apoptotic cells in each section was counted and expressed in millimeters-squared. The number of apoptotic cells occurring within islets was also counted and these results expressed per islet. Serial sections were taken to determine the extent of pancreatic insulin staining. Pancreatic sections were incubated with guinea-pig anti-insulin antibody, followed by a peroxidase-conjugated secondary antibody and DAB. Each section was scored according to an arbitrary scale as follows: $0=$ no insulin-positive cells; $1=1-10$ positive cells; $2=11-20$ positive cells; $3=$ more than 20 positive cells. All sections were scored in a blinded fashion by a single observer (FJL). The results were analysed using Kruskal-Wallis analysis for non-parametric data and were significant when $p<0.05$.

\section{Results}

The incidence of diabetes in the diabetes-prone group of rats was $80 \%$, with a mean age at onset of $83.6 \pm 12.2$ days. No apoptosis was detected in any section obtained from DR animals at any age investigated. Apoptosis was not detected either in any of the pancreatic sections from diabetes-prone and ONDDP animals at 39 and 50 days of age. At 68 days, however, apoptotic cells were detected in $48 \%$ DP and $33 \%$ OND-DP sections and the mean number of apoptotic cells/section area was threefold higher in the DP group than in the OND-DP group. The DP group showed a further increase in the numbers of apoptotic cells at 85 days ( $p<0.05$ vs DR group) but by 107 days the number of apoptotic cells was not significantly different from the DR group. In contrast, amounts of apoptosis in the OND-diabetes-prone sections were significantly higher at 107 days $(p<0.05)$ than in the DR group (Fig. 1).

Apoptosis within islets was detected in both the diabetes-prone and OND-diabetes-prone groups at 68 days. There was a sixfold increase in apoptosis in islets from diabetes-prone animals between 68 and 85 days. At 85 days there was a threefold increase in the number of intra-islet apoptotic cells in the DP group when compared with the OND-diabetes-prone group, although this did not reach statistical significance because 14/24 diabetes-prone sections did not show any apoptosis within islets. At 107 days, the number of apoptotic cells within islets was significantly higher in the OND-DP than in the DR group $(p<0.05)$ (Fig. 2). Immunohistochemical staining for insulin and apoptosis indicates that beta-cells form a proportion of the islet cells undergoing apoptosis in both diabetes-prone animals at the time of diabetes onset (Fig. $3 \mathrm{~A}$ ) and in OND-DP animals at 107 days (Fig. 3B).

The appearance of apoptotic cells at 68 days coincided with the onset of insulitis in both the diabetesprone and OND-diabetes-prone animals [7]. The maximum apoptosis observed in the diabetes-prone group at 85 days also coincided with a significant decrease in insulin staining $(p<0.01$ when compared with the DR group). The OND-DP group also showed a decrease in insulin staining at this time $(p<0.05$ vs DR). By 107 days, however, insulin staining was virtually undetectable in the DP group $(p<0.01$ vs DR) whereas insulin concentrations in the OND-DP group were not significantly different from those of the DR group (Fig. 4). All pancreatic sections from DR animals achieved a maximum score for insulin staining at all time points. 

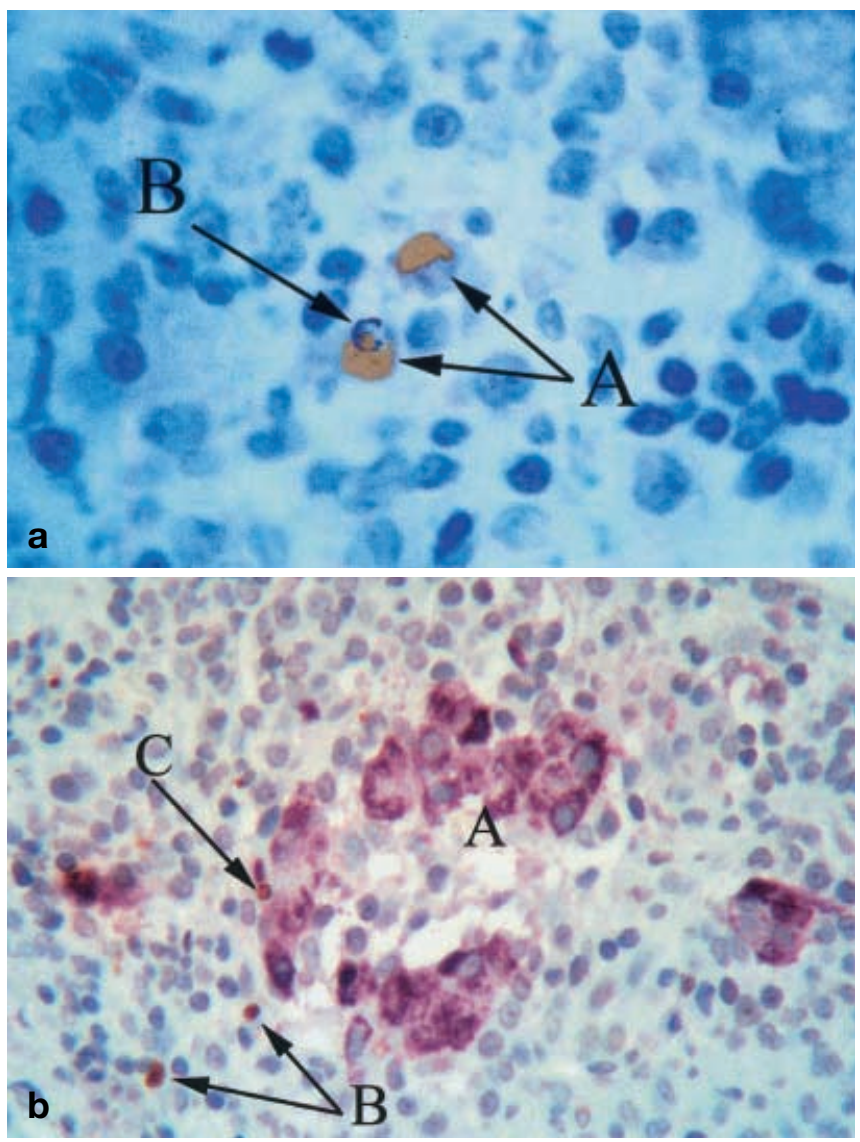

Fig.3A-B. Photomicrographs from immunohistochemical analysis of $\mathrm{BB} / \mathrm{S}$ rat pancreas. (A) Islet from DP rat at time of onset of overt Type I diabetes showing insulin staining (A) and condensation of DNA around the inner nuclear membrane $(\mathbf{B})(\times 400)$. (B) Dual-stained islet from OND-DP rat showing insulin staining (VIP; Vector, UK) (A), apoptotic cells stained with DAB using the Deadend assay (B) and $\beta$-cell with residual insulin which is also positive for apoptosis (C) $(\times 200)$.

\section{Discussion}

Recent experimental data indicate that apoptosis plays a part in the destruction of beta-cells during onset of Type I diabetes [4, 8]. In vitro, apoptosis has been identified as the main mechanism of cell death in islets and beta-cell lines exposed to cytokines and other cellular insults $[3,9]$. The rate of apoptosis and of clearance of apoptotic cells in vivo is such that the mechanism of beta-cell death leading to diabetes still needs to be clarified. The present study shows that apoptotic cells are present in the pancreas of pre-diabetic and diabetic BB/S rats. Apoptosis was not detected in the two earliest pancreatic biopsies (39 and 50 days) but by 68 days apoptosis was detectable in both the DP and OND-DP groups. The appearance of apoptotic cells within the pancreas and, indeed within islets, of DP-BB/S rats at 68 days of age coincides with an increased infiltration of T cells and macrophages [7]. Furthermore, maximal apoptosis in this group occurred at 85 days and coincides with the on- set of diabetes $(83.6 \pm 12.2$ days). It is noteworthy that insulitis was detected in most diabetes-prone and OND-DP biopsies from 68 days onwards but was not observed in any of the biopsies from DR rats [7]. These results are in agreement with studies carried out in the NOD mouse [3] where apoptosis was shown to increase with age and to peak immediately before the onset of diabetes. In another study using NOD mice [4], apoptotic cells were detected as early as 3 weeks of age. In contrast with our studies, this early detection of apoptotic beta-cells preceded the appearance of $\mathrm{T}$ cells which, the authors speculated, may have triggered beta-cell destruction and diabetes. Apoptosis of T lymphocytes following an immune assault has been well-documented [10] and this could account for some, if not the majority, of the apoptotic cells observed in pancreatic tissues. Indeed, apoptosis of infiltrating cells could also account for the large increase in apoptotic cells observed in OND-DP rats at 107 days of age. Apoptosis of CD8 + T-cells has been shown to account for the majority of apoptotic cells observed in the diabetic NOD mouse pancreas [3]. This study shows, however, beta-cell apoptosis in diabetic $\mathrm{BB} / \mathrm{S}$ rats at the time of onset of overt disease.

The experiments using NOD mice $[3,4]$ were done on cohorts of animals in which diabetes outcome was not known. The results therefore included both diabetic and non-diabetic mice which would inevitably have influenced the levels of apoptosis observed. Serial pancreatic biopsies make it possible to trace the course of disease progression in individual animals and hence to identify differences, or similarities, between the DP and OND-DP animals. Using this technique, we have not only shown differences in apoptosis levels between the DP and DR rats, but we have also shown differences in the extent of pancreatic and intra-islet apoptosis between diabetes-prone animals which do or do not progress to overt diabetes. The appearance of apoptotic cells in the pancreatic sections coincides with an increase in infiltration of T cells and macrophages [7] and a decrease in insulin staining. In addition, maximal observed apoptosis in the DP cohort also coincides with the mean age at onset of diabetes. These data suggest that the apoptosis observed in the DP-BB/S animals could be sufficient to account for widespread beta-cell destruction and subsequent progression to insulin-dependent diabetes. In addition, the recovery of pancreatic insulin content in OND-DP rats following insulitis could indicate the existence of possible beta-cell defence and repair mechanisms.

This study shows, for the first time, the existence of significant in vivo islet cell apoptosis in the DP-BB/S rat during the process of insulitis and the onset of diabetes. In addition, this study provides an insight into the differences in apoptosis between the diabetesprone and OND-DP rats which could be an impor- 


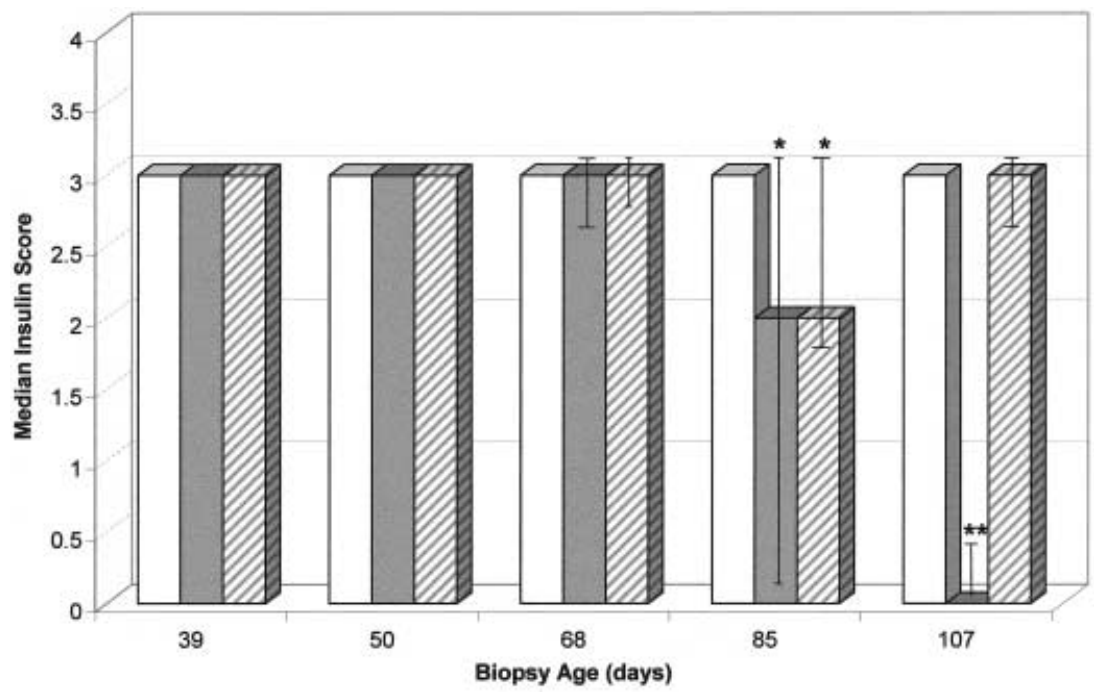

Fig.4. Pancreatic insulin content in DR, DP and OND-DP $\mathrm{BB} / \mathrm{S}$ rats. Serial pancreatic sections from DR $(n=6 ; \square)$, DP $(n=24 ; \square)$ and OND-DP $(n=6 ; \mathbb{Z})$ rats were stained for insulin and scored according to the number of insulin-positive cells in each section. The figure represents the medians of all scores at ages 39, 50, 69, 85 and 107 days. Data were analysed using Kruskal-Wallis analysis and differences deemed significant if $p<0.05$. $* p<0.05, * * p<0.01$ vs DR

tant determinant of disease outcome in this animal model of human Type I diabetes.

Acknowledgements. We are very grateful to L. Dixon for her help with the immunohistochemical studies and to J.Barley for her management of the BB/S rat colony. The financial support of the Wellcome Trust is gratefully acknowledged.

\section{References}

1. Lincz LF (1998) Deciphering the apoptotic pathway: all roads lead to death. Immunol Cell Biol 76: 1-19

2. Mauricio D, Mandrup-Poulsen T (1998) Apoptosis and the pathogenesis of IDDM: a question of life and death. Diabetes 47: 1537-1543
3. Augstein P, Elefanty AG, Allison J, Harrison LC (1998) Apoptosis and beta-cell destruction in pancreatic islets of NOD mice with spontaneous and cyclophosphamide-accelerated diabetes. Diabetologia 41: 1381-1388

4. O'Brien BA, Harmon BV, Cameron DP, Allan DJ (1997) Apoptosis is the mode of $\beta$-cell death responsible for the development of IDDM in the nonobese diabetic (NOD) mouse. Diabetes 46: 750-757

5. Bone AJ, Hitchcock PR, Gwilliam DJ, Cunningham JM, Barley J (1999) Insulitis and mechanisms of disease resistance: studies in an animal model of insulin dependent diabetes mellitus. J Mol Med 77: 57-61

6. Bone AJ, Walker R, Vary AM, Cooke A, Baird JD (1990) Effect of cyclosporine on pancreatic events in $\mathrm{BB} / \mathrm{E}$ rats. Diabetes 39: 508-514

7. Lally FJ, Bone AJ (2000) The in vivo characterisation of insulitis in the spontaneously diabetic BB/S rat. Diabetic Med 17[Suppl 1]: (Abstract)

8. Iwahashi H, Itoh N, Yamagata K et al. (1998) Molecular mechanisms of pancreatic beta-cell destruction in autoimmune diabetes: potential targets for preventive therapy. Cytokines Cell Mol Ther 4: 45-51

9. Delaney CA, Pavlovic D, Hoorens A, Pipeleers DG, Eizirik DL (1997) Cytokines induce deoxyribonucleic acid strand breaks and apoptosis in human pancreatic islet cells. Endocrinology 138: 2610-2614

10. Lenardo M, Chan FKM, Hornung F et al (1999) Mature T lymphocyte apoptosis - immune regulation in a dynamic and unpredictable antigenic environment. Annu Rev Immunol 17: 221-253 